Artículo de investigación

Cómo citar: Campaz, N., y Suárez, N. (2019). Desigualdades de los jóvenes afrocolombianos frente a la educación y la cultura, Polisemia, 15(27), 58-81. http://doi.org/10.26620/uniminuto. polisemia.15.27.2019.58-81

ISSN: $1900-4648$

elSSN: $2590-8189$

Editorial: Corporación Universitaria Minuto de Dios - UNIMINUTO

Recibido: 4 marzo 2019

Aceptado: 6 abril 2019

Publicado:15 mayo 2019

\title{
Desigualdades de los jóvenes afrocolombianos frente a la educación y la cultura
}

\author{
Inequalities of young afro-colombians \\ in the face of education and culture
} Desigualdades da juventude afro-colombiana na educação e cultura

\section{Resumen}

Este artículo presenta la primera parte de una investigación cualitativa llevada a cabo con jóvenes afrocolombianos en tres universidades de Bogotá y con jóvenes mestizos de grado $11^{\circ}$ de un colegio ubicado en los Altos de Cazucá, sobre sus trayectorias hacia la universidad. La idea central es poder presentar las seis desigualdades educativas que enfrentan estos jóvenes frente a la educación y la cultura a partir de la teoría de Bourdieu y Passeron a causa de los condicionamientos sociales. El argumento parte de la idea, de que la inequidad educativa se genera en la educación básica primaria y media para el caso colombiano. Los tres principales condicionamientos sociales se configuran alrededor de la clase social, el espacio y la etnia. Estas tres categorías como instrumentos de conocimiento y comunicación del capital simbólico son establecidas mediante la imposición de distinciones jerárquicas que aseguran ciertos acuerdos sobre el sentido del mundo.

Nubia Suárez Sanabria

Bióloga de la Universidad de los Andes y Maestría en Ciencias de la Universidad Nacional de Colombia. AFILIACION INSTITUCIONAL: Bioestadística SAS. nubia.suarez@bioestadistica.com.co

Palabras clave: desigualdades, racismo, educación superior, espacio, clase social y etnia

\begin{abstract}
This paper presents the first part of a qualitative research carried out with young Afro- Colombians in three universities of Bogotá, and with young mestizos students of the 11th grade of a high school located in Altos de Cazuca.
\end{abstract}


The research deals with an analysis of the students' trajectories towards university. The central idea is to be able to present the six educational inequalities that these young people face to education and culture based on the Bourdieu and Passeron theory because of the social conditioning they face. The central idea is that educational inequality in Colombia is generated on the basis of Primary Education. The three main social factors that influence such inequality are: the social class, the sorrounding space, and the ethnicity. These three categories act as tools for the knowledge and communication of the symbolic capital, and they are established through the imposition of distinct hierarchies that ensure certain agreements on the meaning of the world.

Keywords : inequality, racism, Higher Education, space, social class, ethnicity

\section{Resumo}

Este artigo apresenta a primeira parte de uma investigação qualitativa realizada com jovens afro-colombianos em três universidades de Bogotá e com jovens mestiços no $11^{\circ}$ ano de uma escola situada em Altos de Cazucá, sobre as suas trajectórias em direcção à universidade. A ideia central é poder apresentar as seis desigualdades educativas que estes jovens enfrentam na educação e cultura a partir da teoria de Bourdieu e Passeron por causa dos condicionamentos sociais. $\mathrm{O}$ argumento parte da ideia de que a desigualdade educativa é gerada no ensino primário e secundário no caso colombiano. Os três principais factores de condicionamento social são configurados em torno da classe social, espaço e etnia. Estas três categorias como instrumentos de conhecimento e comunicação do capital simbólico são estabelecidas através da imposição de distinções hierárquicas que asseguram certos acordos sobre o significado do mundo.

Palavras-chave: desigualdades, racismo, ensino superior, espaço, classe social, e etnia. 


\section{Introducción}

$\mathrm{Ni}$ antropólogas ni sociólogas, pero sí con un profundo sentido filosófico que nos lleva a la pregunta por la interpretación de las culturas y la producción cultural. Ya en su libro sobre La Reproducción: elementos para una teoría del sistema de enseñanza, Bourdieu y Passeron argumentan cómo "el llamado mercado de los bienes simbólicos o mercado de los mensajes culturales" (1979, p.16) ocurre en el campo cultural, donde se reproducen las relaciones de clase y se posiciona a la escuela como principal instancia legítima y legitimadora de un arbitrario cultural dentro de la estructura que distribuye el capital cultural entre las clases, gestionando la reproducción de las relaciones entre estas.

De manera particular, Fanon (2010), ha señalado al racismo como una jerarquía de superioridad e inferioridad que ha sido políticamente producida como una estructura de dominación que durante siglos, el sistema colonial, occidental, cristianocéntrico, capitalista, patriarcal y moderno, ha producido sistemáticamente como un "consumo cultural" digno de ser reproducido. En tal sentido, como consecuencia de esta estructura de dominación, las personas entran a ser socialmente reconocidas como humanas o sub-humanas de acuerdo con la posición ocupada en tal jerarquía. Del mismo modo, dependiendo de la posición ocupada, son consideradas en su humanidad como seres con subjetividad y con acceso a derechos.

Ahora bien, en lo que respecta a la producción cultural, la investigación llevada a cabo por Bourdieu sobre el simbolismo, sugiere, que los símbolos son por excelencia instrumentos de la integración social, y en tanto que son instrumentos de conocimiento y de comunicación, hacen posible el acuerdo en la manera de percibir el mundo social, y que contribuye así a la reproducción del orden social de manera lógica como condición de la integración moral. Por consiguiente, la manera cómo funcionan los "sistemas simbólicos" dan razón de las producciones simbólicas asociadas a los intereses de una clase, y tienden a ser representados como universales. De donde resulta, que las proposiciones que aquí se presentan asociadas al tema de las desigualdades, se sitúan en el campo educativo y académico para dar cuenta de una "formación social" como la colombiana, que parte de un hecho social histórico como lo fue el colonialismo y de su dimensión etnográfica en el contexto actual. Será preciso mostrar desde el comienzo, que la comprensión que se tiene sobre las desigualdades educativas están directamente relacionadas con factores históricos de las diásporas africanas, y que desde las investigaciones de Rodríguez (2010), corresponderían a una "falla estructural" que el autor desarrolla en su libro Más allá del desplazamiento. Dichas fallas corresponderían según el autor, a las dificultades, vacíos y cuellos de botella para contar con un registro más claro en la caracterización de las razones que llevaron al desplazamiento, teniendo en cuenta, que dicho fenómeno afecta de manera desproporcionada a comunidades negras. Habría que precisar de lo anterior, que este fenómeno aunado a las fallas estructurales históricas, es lo que hace que los miembros de comunidades afrocolombianas se encuentren en posiciones más vulnerables para acceder a derechos como la educación dentro del espacio social considerado en la teoría de los campos de Bourdieu. 
De la diáspora africana: un continuo rezago y desarraigo

Cualquier ser humano que por intuición o por las experiencias vividas intente comprender el mundo se introduce en una de las artes altamente refinadas de la sociedad contemporánea como la literatura, la música o la experiencia del lenguaje literario. Así por ejemplo, citando a Margaret Mead, Bourdieu y Passeron hacen referencia a la forma como se institucionalizan las costumbres en un contexto dado. En particular, muestran los autores que

Entre los indios de Norteamérica, el comportamiento del visionario era altamente refinado. El joven que no había elegido todavía una visión era habitualmente enviado a escuchar los numerosos relatos de las visiones que habían tenido los demás hombres, relatos que describían en detalle el tipo de experiencia que debía considerarse como una verdadera visión y el tipo especial de circunstancia (...) un examen más en profundidad hacia percibir claramente que la visión no era una experiencia mística democráticamente accesible a cualquiera que la buscara, sino un método cuidadosamente mantenido para conservar para ciertas familias la herencia de la pertenencia a la sociedad de los hechiceros. (Bourdieu y Passeron, 1964, p. 12).

Como ya se ha dicho, se trata del acceso a un bien o a un recurso en el campo cultural, como lo es el de la educación superior universitaria en Colombia. No obstante, para situar a los grupos afrodescendientes en este campo y entender de mejor manera sus trayectorias; se hace necesario echar un vistazo a las circunstancias históricas que rodean su estado actual en una sociedad como la colombiana.

Así, Díaz (2001), ha señalado, cómo para los libertos y afromestizos libres, acceder o desempeñarse como sujetos sociales en el campo de la libertad significó inscribirlos en una nueva dialéctica de pensar la dominación y la dependencia en relación con los demás grupos sociales que componen la nación. Por su parte, Lasso (2015) muestra cómo en la era de las revoluciones independentistas la democracia racial no fue ni inevitable ni un legado colonial, puesto que, aunque las ideas republicanas de ciudadanía alimentaron una ilusión de cambio, en la práctica dejaron intactas las estructuras sociales de las relaciones de poder. Así, la idea de la igualdad racial fue instrumentalizada por la élite para conseguir el apoyo de la población negra durante las luchas de independencia.

En efecto, el racismo como herencia de la esclavitud, la discriminación racial, como fruto de un discurso vacío de las nacientes republicas, y el desplazamiento forzado como un fenómeno económico y político que ha configurado una nueva estructura social, ha afectado de manera importante a las personas de ascendencia africana, con la estigmatización de sus rasgos culturales como una forma de "consumo cultural", que condiciona la inclusión plena en materia de los derechos humanos reconocidos en la Constitución Política con el interés de proteger a todas las personas sin distinción de raza, sexo, origen nacional o familiar, lengua, religión, opinión política o filosófica. 
Con base en las investigaciones que señalan que los mecanismos por los cuales se perpetúa la desigualdad en las sociedades modernas tienen que ver con la pertenencia a un grupo particular, elección personal, herencia familiar o cultural ${ }^{1}$. El estudio de caso en los Altos de Cazucá y en las tres universidades de Bogotá con jóvenes afrocolombianos pretende indagar en sus trayectorias hacia la universidad.

\section{Sistema educativo en Colombia: exclusión social y desigualdades}

Las investigaciones sobre el sistema de educación superior en Colombia han subrayado la clase social, ya que el logro académico está determinado por el nivel de ingreso de las familias y por el nivel educativo de los padres. Pero del mismo modo que por la clase social, el sistema educativo tiene un fuerte componente cultural, esto es, de un modelo de enseñanza que se impone y define en relación con unos valores, costumbres, ideologías, prácticas discursivas y creencias, introducidas desde los comienzos de la educación superior.

Como resultado de esta tradición, las actividades de enseñanza se enfocaron en ciertas áreas del conocimiento, y estaban subordinadas a los dictamines del Estado y de la iglesia católica, dando acceso preferencial a un grupo de estudiantes provenientes de órdenes religiosas y familias españolas o criollas con buena posición social. De este modo, el funcionamiento del sistema de educación superior se entiende mejor si se establecen las diferencias sociales y las desigualdades educativas que fundan el sistema.

Atendiendo a la teoría de Bourdieu y Passeron sobre el sistema de enseñanza y los condicionamientos sociales que tienen los estudiantes para relacionarse con la cultura educacional, en el siguiente apartado se abordan seis desigualdades para el acceso de los jóvenes afrocolombianos a la educación superior, lo que muestra que si bien la clase es un factor importante no se pueden subvalorar los particulares efectos del racismo que enfrentan. En efecto, al explorar las percepciones culturales sobre las barreras de acceso y mecanismos simbólicos para el acceso en la educación superior de jóvenes que están terminando la educación básica primaria y media se encontró que algunas de estas seis desigualdades se convierten también en barreras para el acceso.

1 En un artículo titulado Equidad en la diferencia: políticas para la movilidad social de grupos de identidad, Cárdenas, Ñopo y Castañeda (2012) muestran cómo las categorías de etnia, región o clase social se encuentran correlacionadas con las desigualdades entre grupos étnicos. Es decir, que cualquier individuo que se reconozca como perteneciente a alguna de estas categorías se encontraría en total desventaja frente al acceso a oportunidades de bienestar en comparación con otros individuos que dicen no pertenecer a ninguna de estas. 
Desigualdades de los jóvenes afrocolombianos frente a la educación y la cultura

Se suele considerar que todo aquel aspirante que haya cursado la educación básica y media cuenta con los conocimientos para competir en igualdad de condiciones por un cupo para estudiar en la universidad. Es más, se ha creído que el saber por sí mismo como principio de emancipación y avance del ser humano, sin distinción de raza, género, origen social o color de la piel, o que las políticas diferenciales (acciones afirmativas) implementadas por las universidades contribuiría a la desaparición de las desigualdades sociales y de los privilegios (Castón, 1996; Restrepo, 2007; Andrade y Andrade, 2012; Rodríguez, 2016). Sin embargo, algunos analistas de la teoría de la reproducción cultural de Bourdieu y Passeron, señalan, que la Escuela y el sistema universitario, definido tradicionalmente por los sociólogos como "el conjunto de mecanismos institucionales o consuetudinarios por los que se halla asegurada la transmisión entre las generaciones de la cultura heredada del pasado" (1979, p52), reproducen los valores de una cultura, denominada también como la "gran cultura", ya que su función es seleccionar a los estudiantes de las clases más favorecidas y segregar a los estudiantes de las clases populares.

Para Bourdieu y Passeron, la "acción pedagógica" y el "trabajo pedagógico" entrarían a formar parte de aquellos mecanismos por los cuales la escuela como sistema asegura la transmisión de los valores de la cultura de la clase dominante (gran cultura), como dignos de ser reproducidos, pero rechazando y despojando de contenido y privando de significado a los sujetos que la ejercen. Las dos categorías introducidas por los sociólogos son entendidas también como "juicio profesoral" o "juicio experto" con el cual se clasifica a las categorías de estudiantes en una determinada formación social (Subirats, 1979; Bechelloni y Ciafaloni, 1979; Canclini, 1985; Gutiérrez, 2011).

De acuerdo con Bechelloni y Ciafaloni (1979), Bourdieu y Passeron sostienen la tesis, de que el sistema escolar tiene una autonomía relativa en cuanto a la transmisión de la enseñanza, ya que sirve de manera específica e insustituible a las estructuras sociales. Conviene subrayar, que el objeto de análisis es el campo cultura-mercado de los bienes simbólicos o mercado de los mensajes culturales, donde tiene lugar la producción de significados jalonada por sujetos concretos en un sistema de relaciones de fuerza entre los grupos o clases sociales.

Al tratar de establecer cómo estos condicionamientos sociales reproducen la desigualdad entre las clases, se advierte, que la "ideología carismática" convierte la herencia social en mérito personal, encarnando el talento en el "niño prodigio" para justificar lo arbitrario del privilegio (Bourdieu y Passeron, 1964). Así, los condicionantes sociales por las carencias acumulativas padecidas, por los grupos afrodescendientes dada la estructura racista, más la discriminación racial y el desplazamiento forzado, son convertidos en "deficiencias mentales", "deficiencias comportamentales" o "desvia- 
ción cultural" en los estudiantes por el discurso de la igualdad formal, que considera, que todo aquel "aspirante" que haya cursado la educación básica y media debe contar con los conocimientos para tomar el examen de admisión, sobre todo en la Universidad Nacional de Colombia, más allá de las características que condicionan la inclusión plena dentro del sistema².

De manera que, el presente artículo tiene como finalidad presentar los hallazgos de una investigación cualitativa realizada con jóvenes afrocolombianos universitarios en tres universidades públicas de la ciudad de Bogotá y con jóvenes de grado $11^{\circ}$ de un colegio ubicado en la zona de los Altos de Cazucá por medio de entrevistas en profundidad, grupos focales y observación participante, complementadas con entrevistas estructuradas a expertos en etnoeducación. Al caracterizar las seis desigualdades sociales se pretende indicar cómo estas devienen en barreras para el acceso a la educación superior universitaria y se reproducen culturalmente de generación en generación en las prácticas discursivas.

\section{Desigualdad ante la cultura}

Morales (2008), citando a Taguieff, señala, que el racismo como una ideología cuyo núcleo es la desigualdad basada en la diferencia de naturaleza, ocurre en las prácticas de exclusión, acompañadas de odio y desprecio del otro al concebir la diferencia como negativa o de difícil consenso. Por su parte, Reygadas (2008), muestra, cómo en la mayoría de los países latinoamericanos, a los factores económicos y políticos se han unido consideraciones étnicas, lo que genera una mayor inequidad social. Es así como aparece un tipo de "desigualdad reforzada", por las categorías internas que dividen a un grupo, por ejemplo patrones-trabajadores, jefes-empleados, dirigentessubordinados, entre otras, se organizan con categorías externas como negroblanco, mulato-blanco, mulato-negro, indígena-europeo indígena-mestizo y mestizo-blanco, construidas en el periodo colonial. Con el paso del tiempo estas categorías pierden su valor legal, no obstante se reprodujeron en la vida cotidiana de una manera más borrosa.

2 En un artículo titulado La sociología de la cultura de Pierre Bourdieu, Néstor García muestra, que las preguntas fundadoras de casi todos los trabajos de este autor, aunque nunca las plantea directamente, tienen que ver con cómo están estructuradas económica y simbólicamente la reproducción y la diferenciación social; cómo articulan lo económico y lo simbólico en los procesos de reproducción, diferenciación y construcción del poder. Aunque su respuesta se enfoca en mostrar la estructura de las clases sociales como relaciones de lucha, su teoría social, dice García, incorpora otros aspectos dedicados a estudiar los sistemas simbólicos y las relaciones de poder que para Bourdieu significa romper con el pensamiento lineal, que no reconoce más que las estructuras de orden simple de determinación directa, y tratar de reconstruir en cada investigación las redes de relaciones en las cuales se apoyan distintos factores de desigualdad. 
Se infiere en que, esta manera borrosa es, quizás lo que algunos analistas han denominado como formas eufemizadas de racismo definidas por ingresos, el estilo de vida, el consumo cultural, la escolaridad y la calidad de la educación recibida ${ }^{3}$. Es preciso indicar, que en el origen de la desigualdad en América Latina se encuentra la conquista europea de las poblaciones indígenas, el trauma de la esclavitud negra y la concentración de la propiedad de la tierra. Aunque la teoría sociológica de Reygadas sobre La Apropiación hace ver, cómo en los países latinoamericanos la clase dominante es predominantemente blanca, frente a la mayoría de la población que no lo es, el trabajo etnográfico realizado en la presente investigación muestra claramente la intersección entre clase, grupo étnico y género:

Nuestro país más que racista, es clasista. Porque lo que decían hace un rato: una frase que no recuerdo bien quién la dijo, y es que cuando ven la hoja de vida que tienes y ven quién tú eres, hasta se les puede olvidar el color. No es tanto así como que en serio se les olvide. Pero si es que lo olvidan y lo pasan a un segundo plano, porque entonces empieza a importar más vos quién sos. Entonces es la gran doctora, el gran doctor, el señor o la señora. Entonces ya no es el negro o el blanco, si no es el que tiene la plata. (Entrevista grupo focal estudiantes del programa Martin Luther King Jr., 2016).

La percepción respecto de que "nuestro país más que racista es clasista" tiene que ver con la categoría acuñada por Tilly Reygadas (2000) sobre "desigualdad reforzada", cuando a los factores económicos y políticos se han unido consideraciones étnicas que hacen que la inequidad entre los grupos sociales se haga mucho mayor. Ahora bien, la pregunta por el ¿vos quién sos?, que corresponde a las atribuciones "gran doctora" "el gran doctor" "el señor" o "la señora", que como señala Reygadas son construidas mediante dispositivos sociales, económicos, políticos y culturales que separan, marcan diferencias, y del mismo modo, favorecen a la distribución asimétrica de la riqueza, el poder, el prestigio y el bienestar.

De otra parte, la diferencia genética como último refugio de las ideologías racistas, fruto de la categoría raza producida discursivamente organizando rasgos asociados a la manera de hablar, las representaciones y las practica sociales, utiliza características físicas como el color de la piel, la textura del pelo, los rasgos físicos y corporales como marcas simbólicas con la finalidad de hacer la diferencia entre grupos o clases (Restrepo, Walsh y Vich, 2010).

3 En el diccionario de la Real Academia Española (RAE) la palabra eufemismo se traduce como "manifestación suave o decorosa de ideas cuya recta y franca expresión sería dura o malsonante". Al analizar el artículo sobre el racismo de la inteligencia, Néstor García muestra, que el racismo elevado a un alto grado de eufemización se enfrentaría a problemas de comunicabilidad lo que le llevaría a declararse en favor del eugenismo. Entre tanto Roxana Kreimer al realizar un análisis sobre la historia del mérito muestra, que en la episteme de la modernidad, la eugenesia surge con base en la teoría darwiniana sobre la selección natural, en la cual, la lógica de la eugenesia no es ajena a ciertos rasgos de una meritocracia empeñada en discriminar a los individuos competentes de aquellos que no lo son. 
Que ves también: con un profesor tuve un rose. Él estaba dando un ejemplo, de los atletas, y él decía que las personas afro son muy buenas para el deporte. O sea, yo lo digo afro porque suena mejor que decir una persona de color. Porque no somos de color, somos afro o quizás tengamos raíces palenqueras, somos negros, en fin. Entonces él dijo las personas de color tal cosa... Entonces una compañera atrás se echó a reír. Entonces yo le dije: profe, puedes decir personas palenqueras, personas negras, personas de raíces africanas, afrodescendientes, pero no vuelvas a decir de color porque se escucha mal y a mí no me gusta. Si se escucha mal y me ofende. $\mathrm{O}$ a veces, cuando me he venido con el cabello suelto, mi cabello es afro. Y empiezan a tocarlo: iay pero tu pelo cómo es! ¡Ay pero no me lo toquen! (Risas) O sea como que en muchas áreas, las personas son así. $\mathrm{O}$ a veces, porque uno habla ve, ¿y vos qué? ¿Por qué estás así? Las personas no entienden que uno viene de otra parte, si no que venga, hágame el favor y tal cosa. Pero nosotros somos de otra parte. Entonces vamos a tener un acento diferente. Como que vení, hacete pa'ca. O sea, son cosas que debemos entender, ¿'sí? Entonces todas esas cosas me han llevado a esquivarme un poco, la verdad. Porque me han hecho sentir muy incómoda. (Entrevista a la estudiante Quiñones, 2018).

En principio habría que decir, que existe una desigualdad basada en la diferencia, que como indica Reygadas, se ha construido en el periodo colonial a partir de la discriminación étnica y de relaciones de poder. En realidad como señala Hall, el discurso como hecho social construye la subjetividad para que cada persona o grupo produzca y reproduzca una representación social sobre sí mismo y sobre los demás (Restrepo, Walsh y Vich, 2010). Como también lo ha argumentado Díaz (2001), la esclavitud como institución de la marginalidad, hoy se perpetúa en una serie de situaciones y condicionamientos sociales que tienen las personas ascendencia africana, para que sean incluidas plenamente en la vida social.

\section{Desigualdad ante la educación}

Al hablar de la producción social de individuos desiguales, Reygadas, señala, que esta ocurre desde las diferencias entre personas hasta las grandes asimetrías globales. Así por ejemplo, si alguien alcanza un desempeño escolar impresionante, que después le lleva a obtener un buen trabajo y grandes ingresos, es algo que no depende solo de sus genes o de su dedicación al estudio, sino también de la nutrición propia y de la de sus padres, del capital académico y cultural que adquirió en el seno familiar, de la calidad de sus profesores y de sus escuelas, de las redes sociales en que se movió Reygadas (2008). Así que Nery, al hablar de sus dificultades en el colegio y en la universidad argumenta que:

Económicamente pues, era el tener que llegar a la casa y no había qué comer. Y uno a pie, con sol, con hambre, y uno tenía que hacer la tarea y no había qué comer. Y esperar a que llegara el papá y a comprar la aguapanela con arroz con papa y eso hasta el otro día a ver si aparecía. Bueno, hasta que uno llegaba a la casa y se acordaba que no había que comer. (Risas). (Entrevista a la estudiante Palma, 2018). 
Para precisar esta narrativa, Rodríguez, Alfonso y Cavelier (2009) en el informe sobre Raza y derechos humanos en Colombia muestra, cómo en materia de pobreza, hambre e indigencia, las diferencias son considerables entre grupos étnico-raciales. Básicamente, la afectación de este fenómeno sobre la población afrocolombiana se da en el contexto de una extrema marginalidad económica como también apunta Nery. Por lo demás, la desigualdad educacional se muestra más impiadosa, si como se ha dicho, las investigaciones sobre el logro educativo en Colombia están asociadas tanto al nivel de ingreso de las familias como al nivel educativo de los padres. Así las variables de entorno ${ }^{4}$ asociadas a los estudiantes permiten ver la profunda desventaja generadas por las mismas

De hecho yo, en Timbiqui me limitaron mucho, yo volaba mucho de aprendizaje. Entonces allá no habían recursos, no habían libros, unos libros que tenían los profesores, entonces yo buscaba donde aprender más y no había. Hasta que una vez un tío mío llevó una enciclopedia y yo me aprendí esa enciclopedia completica [...] pero yo ya era grande, ya viejo, si hubiese salido más niño, hubiera desarrollado más la inteligencia, pero ya salí muy adulto. Ahí está el detalle, a mí me hubiese tocado más duro acá en Bogotá, en cuanto a la tecnología y a muchas cosas, yo iba muy atrás. Pero muchas veces yo sentía que iba muy adelante acá con los compañeros, en el tema de la matemática, de la física, de cultura general, yo iba muy adelante, en ese sentido. $O$ sea, sabia cosas que personas de la ciudad no la sabían y que pasaban acá en la ciudad, pero era porque tenía esa enciclopedia y yo todo el tiempo estaba ahí, ahí pendiente, veía noticias. (Entrevista al estudiante Loango, 2018).

Como ha señalado Reygadas, la producción social de individuos desiguales es histórica, y tiene que ver con un aspecto del poder: individual, relacional y estructural con connotaciones específicas en cada caso. Así por ejemplo, las capacidades y potencialidades individuales, de acuerdo con este autor, tienen que ver más con las características del individuo y el control que esto pueda tener sobre ciertos recursos, pero que de todos modos están insertas en relaciones de poder.

\section{Desigualdad dentro de la estructura familiar y atmosfera intelectual del medio familiar}

Reygadas (2008) muestra que, en las interacciones asimétricas, donde las personas se unen y se relacionan, se genera también una desigualdad importante a saber, que la dinámica de los campos incide sobre los mecanismos de distribución de las riquezas generadas colectivamente. Tanto el tamaño como las características de las familias, entendidas estas como unidades

4 El estudio que ofrece Melo y otros (2014) sobre la situación actual de la educación superior en Colombia y el análisis de eficiencia, muestran que las variables de entorno, tales como el ingreso de los hogares, el nivel de formación de los padres, la infraestructura, las relacionadas con el personal docente, entre otros, cumplen un rol importante en la explicación de los logros obtenidos por los estudiantes y las instituciones de educación superior. 
económicas, reproducen condiciones asociadas con la desigualdad, puesto que puede existir una desproporción entre productores y consumidores dada las relaciones entre el género y las generaciones.

Al principio fue un poco complicado, mi mamá nos mandaba muy poquita plata para estar el mes y tú sabes que a uno en derecho le toca leer muchísimo, que las copias, que no sé qué... pero bueno, yo eso siempre lo vi bien, pues yo dije: mi mamá no tiene, tampoco uno la puede obligar, está haciendo un esfuerzo grande para que uno esté estudiando. Ya después, bueno en ese tiempo también estaba estudiando mi hermana, mi hermano y yo, éramos tres, mi hermano en una privada en Medellín, mi hermana acá y yo también; y mi mamá es madre soltera. (Entrevista a la estudiante Naysir, 2018).

De otra parte, Melo, Ramos y Hernández (2014) han mostrado como las variables de entorno inciden de manera significativa en el logro académico de los estudiantes. Precisamente, tanto Rodríguez, Alfonso y Cavelier (2009) como la Procuraduría (2006) han mostrado como la población afrocolombiana presenta altos niveles de analfabetismo, lo que significa que las niñas y los niños no cuentan con un entorno intelectual favorable como referente motivacional para el ingreso a la universidad.

Aunque por las situaciones que se presentaron en el municipio, pues mi abuela no es estudiada, ni nada de eso. Pero en cuanto lo que ella podía ella me ayudaba. Y pues yo buscaba apoyo en los profesores. Yo salía de clase y, pues no entendía este ejercicio o lo que sea, yo me iba a la casa del profesor que me explicara, y pues así adelantaba bastante. (Entrevista al estudiante Caicedo, 2018).

\section{Desigualdad por condicionamientos institucionales}

La inequidad tiene una dimensión simbólica, puesto que en sí misma la diferenciación categorial no produce una desigualdad profunda y persistente (Reygadas, 2008). La diferenciación categorial viene aunada con la jerarquización: límite bien definido que separa a sitios con flujos de recursos que sostienen su interacción. De tal manera que los bienes, los recursos, las personas y los conocimientos no circulan libremente; esto es, que siguen una orientación cultural, atendiendo a relaciones de poder y sujetos a constreñimientos institucionales. Maryi ilustra este punto:

Pues mi proceso de ingreso fue, quería entrar a la Nacional también, pero no pude. Entonces una amiga mía, vino pues y me dijo que por qué no entraba a la Distrital. Entonces ella me dijo que fuera a unas charlas que estaban haciendo en una de las facultades. Entonces yo me acerqué directamente a las charlas, estuve todo pendiente, entonces pues allá me dijeron que si aspiraba entrar a la universidad, el Icfes, el puntaje que tenía que tener y que no me garantizaban que yo fuera a entrar solo porque yo había ido a las charlas, solo que tenía que buscar, pues los papeles que tenían para entrar. Entonces hablaban sobre un código y podían entrar por racismo, desplazados, o por afrocolombianidad o indígenas. Entonces yo decidí entrar por ese decreto y entonces yo llevé todos mis papeles. Pues en lo de los papeles tuve como dos dificultades porque me 
pedían una estratificación económica y pues no sabía cómo buscarla. Y pues a pesar de que en la universidad decían cómo era, pero yo no pude. La otra, un certificado que pedían del colegio y pues justamente no me lo daban. Y la tercera es que me pedían una historia clínica y en la EPS no me la daban a mí, sino que se la daban a mi mamá y como ella no podía faltar esos días al trabajo. Entonces tuve pues a punto de perder mi cupo en la universidad. Y ahí pues, fue algo muy duro, porque al no tener esos papeles o sea, no guardaban cupos. $\mathrm{O}$ sea, si no tienes los papeles, pues te quedas fuera. Entonces, pues, fue algo muy duro. (Entrevista a la estudiante Campaz, 2018).

Si gran parte de la capacidad educativa individual de una persona puede aumentar o disminuir por los condicionamientos de entorno, esta misma capacidad se ve disminuida por los constreñimientos institucionales, puesto que como señala Reygadas, para acceder a los recursos acaparados, los individuos se ven obligados a pagar una cuota por haber sido excluidos de estos.

\section{Desigualdad por ubicación geográfica}

Otra de las desigualdades que se asocia con variables étnicas y raciales tiene que ver con la separación espacial y geográfica. Así, las élites se encuentran alejadas espacialmente del resto de la población. En parte, las diferenciaciones se enmarcan en aspectos relacionados con la carencia de infraestructura mínima de comunicaciones, la distancia educativa, que distingue a quiénes han estudiado posgrados o quiénes han ido a las mejores universidades, quienes no saben leer ni escribir, quienes hablan muy bien inglés de quienes apenas conocen algunas palabras, quienes se conectan de manera cotidiana y rápida a Internet, de quienes no tienen acceso a este.

Por tal razón, cuando se trata de ver los alcances de la dimensión simbólica de la desigualdad, se encuentra que también la misma dimensión de humanidad es cuestionada. Así, de acuerdo con Reygadas (2008), los rasgos físicos, sociales y culturales de las personas son inferiorizados en una sociedad donde los grupos que la conforman se ingenian caminos para distinguirse de otros, sustentando tales percepciones en designios de pureza. William contribuye a observar algunas de las formas de producción de estas distancias espaciales o geográficas en el contexto colombiano.

En cuanto a las comunidades, no solamente del Pacifico, no solamente de comunidades negras, sino otras comunidades en Colombia, porque igual Colombia es un país de diversidades. Pero enfocándome en la zona del Pacifico, principalmente en mi comunidad, pues la educación allá no es que sea como uffff, la madre en educación, no. Pues los jóvenes perdemos mucho, bueno en este momento, los jóvenes pierden mucho tiempo, igual yo también perdí tiempo en cuanto a que lo acostumbran mucho a trabajar a uno [...] creo que no solamente pasa en mi comunidad, sino en los municipios aledaños al mío; que pues los jóvenes van, estudian. Y pues de allí les toca salir a trabajar, les toca irse a pescar o a lo que sea, porque igual toca conseguir recursos y esa es una desventaja que tenemos frente a los demás. Porque igual es como lo que aprendemos en el colegio. No sacamos el tiempo como; bueno no es que no 
saquemos el tiempo, sino es que no lo tenemos. Como para, bueno, yo miré este tema en el colegio, lo voy a investigar en mi casa. Y pues eso acá es una ventaja porque llegan los jóvenes en el colegio le dan un tema y llegan, pues a la casa, tienen un papá que es profesional, que tiene una buena carrera y le dice: no pues mira, podemos hacer esto, esto y esto y averiguamos tal cosa, y aprende. Igual allá se aprende mucho, demasiado. Pero pues, en cuanto a eso no se tiene nada. Entonces se está en desventaja. (Entrevista al estudiante Caicedo, 2018).

En el misterio de la desigualdad persistente en América Latina, Reygadas aborda el tema de las distancias físicas, simbólicas y emocionales entre las élites y el resto de la población para referirse, a que la distancia espacial creada entre los grupos sociales no es al azar, sino que tiene una historia política y cultural. Así, para los negros que huyeron hacia los quilombos o palenques, en el caso colombiano, para no ser esclavizados quedaron en el abandono y olvido por parte del Estado y sus instituciones. En consecuencia, dada la distancia física generada entre los grupos sociales, la lejanía geográfica, la desconexión de las nuevas tecnologías y la inserción precaria a los flujos de riqueza por la segregación racial, la inequidad educativa en este caso se hace mucho mayor.

\section{Desigualdad por las condiciones materiales y sociales de existencia}

Este tipo de desigualdad tiene un origen histórico. Sus orígenes históricos se remontan, según Reygadas (2008), en la conquista de los pueblos indígenas, el trauma de la esclavitud negra y la concentración de la propiedad de la tierra. Tanto indígenas como negros fueron integrados de manera desigual, configurando una matriz social con importantes diferencias en términos de estatus, poder y recursos económicos. En esta empresa conquistadora, los colonizadores, además de buscar obsesivamente metales preciosos, también se reservaron las mejores tierras, que posteriormente forjaron y perpetuaron sociedades muy inequitativas.

En una sociedad como la colombiana organizada en torno a castas, como sugiere Rodríguez, Alfonso y Cavelier (2010), el status como una ideología aristocrática continúa distribuyendo bienes y recursos de manera desigual entre los distintos grupos sociales. Así, los criterios por los cuales la sociedad colombiana perpetúa las prácticas colonialistas de discriminación racial se configuran alrededor de méritos escolares, los ingresos, la religión o la nacionalidad, el estilo de vida y el consumo cultural (Moreno y Ramírez, 2003).

La observación de Eliana Fernanda, contribuye de mejor manera a iluminar como se replican estas prácticas

Se escogieron unos perfiles de las personas, y alguno les poníamos el mismo perfil, les poníamos una foto de una persona afrodescendiente y una persona blanca, y estamos pendiente a cuál de las dos personas llamaban más, siendo el mismo perfil. Se enviaban hojas de vida entre hombres y mujeres, y diferentes tonalidades de afrodescendientes, porque eso también influye mucho. Se evidenció, que efectivamente entre menos negro fuera, pues te 
llamaban, no. Entre más bonita fueras, más opciones de que te llamaran tenías. Y también el nombre, entre más menos étnico, había también una posibilidad de que llamaran más. Ese fue como el experimento. Y también pasamos derecho de petición a algunas entidades del gobierno y a algunas entidades privadas, especialmente medios de comunicación, preguntándoles sobre el número de afrodescendientes que tenían contratados y en qué cargos. Entonces ese fue ya un indicador. Primero porque muchas de las entidades públicas, pues no tenían esos indicadores. Sino que decían, que ellos no lo incluían a la hora de contratar personas que porque eso era un acto de discriminación. Entonces nosotros decíamos, no, no es discriminación, por el contrario, ustedes deberían dar esa información porque es un factor de inclusión de la población afrodescendiente. Otro, por ejemplo el de la policía fue muy chistoso. Llamaron de la policía al observatorio diciendo, "es que lo que ustedes me piden es que yo saque a todos los negros y los cuente. Y mi compañera le contestó": "pues sí, básicamente sí, y dígame en qué cargo están”. Y entonces él decía: "pues no, es que eso es mucho trabajo, y eso está como mal, ¿no?” Y mi compañera le explicaba el por qué, y finalmente, el dato nunca nos lo mandaron. La mayoría de los derechos de petición fueron respondidos como que: "nunca habían tomado en cuenta ese indicador". Muy pocos nos contestaron, como que tenemos dos o tres personas en tales cargos. También hay casos específicos que también nos han llegado como actos de discriminación. Esos también nos ayudaban a ver, qué tipos de discriminación sufrían las personas afro en los empleos. Entonces ellos hacían mucha referencia a tratos despectivos, al uso del negro para todo lo que se le fuera a decir, el negro como un apodo, como un problema, siempre señalando sus fortalezas en cuanto a la fuerza, pero no en cuanto al conocimiento. Que si se presentaba alguna queja al interior del trabajo, que la persona afro se había sentido discriminada, entonces se los encasillaba que se sentían perseguidos, que sentían discriminación en todo lado. En el grupo focal, conversando con las psicólogas, ellas nos decían, que específicamente, algunas empresas nos dicen que no quieren gente negra. (Entrevista a experta Antonio, 2017).

Considerando los argumentos de Reygadas acerca de los factores económicos y políticos se han unido circunstancias étnicas que generan una mayor inequidad social; habría que decir, que las desigualdades señaladas se circunscriben en un contexto de relaciones de poder apuntaladas por la estratificación o jerarquización de las categorías de espacio, clase y etnia estigmatizadas. A la narrativa de Eliana Fernanda se une la de Juan de Dios para mostrar las limitaciones de acceso a las oportunidades educativas y de otro tipo.

Para eso debemos tener presente la historia. Y lo primero que hay que tener presente es, que la gente africana en la condición de esclavo, en la condición de no persona, no tenía acceso ni derecho, si quiera a saber leer y escribir. Era prohibido. Cuando llegamos a la ley sobre la libertad de los esclavos, no se reconoce calidad de persona y de ciudadano a las personas africanas liberadas. Así se habla de la libertad de los esclavos que es un contrasentido. Por tanto, no les reconocen, ni tierras, ni educación, ni pago de reparación, ni ciudadanía, ni trato de persona. Solo siguen llamándolo negro. (Entrevista a experto Mosquera, 2018). 
Para tratar de contextualizar la motivación que nos lleva a la pregunta por la interpretación de las culturas y de la producción cultural en el contexto colombiano, los aportes de Reygadas son bastante claros y se conectan con los de Bourdieu y Passeron sobre el llamado mercado de los bienes simbólicos o mercado de los mensajes culturales. En su libro La Apropiación: destejiendo las redes de la desigualdad, Reygadas, muestra, cómo la inequidad tiene una dimensión simbólica. Así por ejemplo, sería muy fácil entender la desigualdad social atendiendo solo a las capacidades individuales. Sin embargo, los bienes, las personas, los recursos y el conocimiento no circulan libremente. Para este sociólogo, lo anterior, tiene que ver con relaciones de poder, orientaciones culturales y constreñimientos institucionales.

Citando los estudios de Norbert Elías en una pequeña comunidad de clase obrera en Londres, Reygadas permite ver, cómo ocurren los procesos de estigmatización con los considerados forasteros. Así, los miembros de un grupo establecido se presentan como mejores seres humanos que el resto, estableciendo tabúes para limitar el contacto y se apropian de los puestos dirigentes en organizaciones locales. Del mismo modo, citando a Abner Cohen, Elías, muestra, el estudio sobre los rituales de exclusividad que permitían a un grupo étnico preservar sus privilegios sociales, políticos y culturales (Reygadas, 2008).

El primero de los cinco postulados que propone Reygadas, como punto de partida del enfoque de la apropiación-expropiación para analizar la desigualdad social, permite ver la existencia de mecanismos de apropiación, por los cuales los individuos o colectivos organizan los beneficios diferenciales y acceden a porciones asimétricas de la riqueza y el bienestar social, y del mismo modo, las cargas y desventajas están distribuidas de forma dispareja.

La nueva dialéctica propuesta por Díaz (2001), para pensar la dominación y la dependencia respecto de los demás grupos sociales en el contexto colombiano tiene que ver, a mi modo de ver con el "paternalismo lastimero" sugerido por Fanon, Bourdieu y Passeron, al mostrar, por un lado, que los negros continúan conservando la condición de salvajes o bárbaros, impermeables a la lógica y a las ciencias. Por otro lado, el "brahmán que da nacimiento espiritual y enseña cuál es el deber de los hombres" encarnado en la acción pedagógica y la autoridad pedagógica profesoral, viene a sustituir, para los grupos afrocolombianos infantilizados, la autoridad paternal y moral que transfiere cosas y reparte cosas, esto es, los bienes codiciados.

\section{Discusión}

Al determinar y caracterizar las seis desigualdades ante la educación y la cultura, no nos proponemos hacer un análisis comparativo; por lo menos no entre los estudiantes afrocolombianos y no afrocolombianos, que han ingresado en tres universidades en la ciudad de Bogotá, pero sí sugerir las diferencias posibles entre estudiantes provenientes de los grupos poblacionales que componen la identidad cultural colombiana. 
Es importante resaltar, que estas desigualdades son tomadas del análisis que hacen Bourdieu y Passeron (1997) en su libro Los Herederos: los estudiantes y la cultura, sobre los condicionamientos sociales que tienen los estudiantes para relacionarse con la cultura educacional. Luego en La Reproducción: elementos para una teoría del sistema de enseñanza, estos sociólogos ponen el acento en el cuerpo docente y en la escuela como sistema para indicar que su autoridad sea legitima por "la institución investida de la función social de enseñar y de lo que es legítimo aprender”.

Cuando decimos que no es un análisis comparativo entre estudiante es porque, no estamos haciendo análisis sobre los índices de acceso, permanencia y graduación entre estudiantes, sino describiendo y explicando los condicionamientos sociales que tienen los estudiantes para acceder al sistema educativo dentro de las posibilidades de acceso. Ahora bien, de acuerdo con Rodríguez (2016), las políticas de acciones afirmativas para el acceso en la educación superior de grupos étnicos son de carácter reciente, lo que significa que cualquier estudio comparativo debería dar cuenta del impacto por acceso diferenciado de los distintos grupos sociales en el contexto colombiano.

Ahora bien, si la conclusión de Díaz es la de pensar a las personas de ascendencia africana como sujetos sociales en el campo de la libertad, lo cual representa y significa ingresar a una nueva dialéctica de pensar la dominación y la dependencia, entonces para comprender realmente lo que va a significar posteriormente la ambigua y diferida libertad no es más que la dominación y la sujeción a una autoridad paternalista que la clasificará y la jerarquizará de por vida en el espacio y tiempo. Para entonces la pregunta que puede surgir es, ¿cómo surgirán las categorías con las que los jóvenes afrodescendientes van a ser clasificados?

¿Quiénes tendrán la autoridad para hacerlo? Y al mismo tiempo, ¿quién les ha dado tal autoridad? ¿Con qué finalidad se hará tal clasificación y jerarquización? Estas podrían ser algunas de las preguntas a plantear, al tiempo que las aportaciones de Bourdieu sobre el poder simbólico ${ }^{5}$, nos conduce a pensar por una parte, en las condiciones que hacen posible las producciones simbólicas como instrumentos de conocimiento y comunicación, y por otra, como instrumentos de imposición o de legitimación de la dominación, ya que cumplen una función política.

5 Para Bourdieu, los sistemas simbólicos como por ejemplo, arte, religión y lenguaje son instrumentos de conocimiento y comunicación que, al tiempo que cumplen una función política cumplen también una función gnoseológica, es decir, de instrumentos de imposición o legitimación de la dominación que contribuye a asegurar la dominación de una clase sobre otra (violencia simbólica) para imponer la definición del mundo social, más conforme a sus intereses, por ejemplo, el campo de las tomas de posición ideológica, ya sea a través de las luchas que libran los especialistas de la producción simbólica dice Bourdieu o por el poder de imponer e inculcar instrumentos de conocimiento y de expresión, taxonomías arbitrarias de la realidad social. 
Si la producción de categorías como espacio, clase y etnia, como categorías de diferenciación y jerarquización nos parecen tan obvias y evidentes, es porque de manera consciente o inconsciente contamos con las disposiciones corporales y mentales para aceptar como una doxa ser diferenciados y espacialmente situados en relaciones sociales y políticas que a nuestro modo de ver no hacen más que confirmar el racismo de la inteligencia, que como esencialismo dice Bourdieu (1997) es una forma de sociodicea característica de una clase dominante, cuyo poder reposa en parte sobre la posesión de títulos, y ya que como estos son garantía de inteligencia se puede hacer uso de ellos para acceder a las posiciones de poder y de propiedad para marcar las diferencias.

En tal sentido como lo señala Restrepo (2007), refiriéndose a las llamadas acciones afirmativas como "políticas de discriminación positiva" hay que pensar estas acciones como entramados sociales más allá de los actos de buena voluntad y reflexividad para cuestionar y cuestionarse frente a la imposición de categorías de pensamiento jalonadas por las universidades mismas y sus intelectuales; para excluir a quienes hay que excluir y para continuar con la dominación cultural convertida en la actualidad como colonización mental o colonialismo interno para citar a Reygadas ${ }^{6}$

En tal caso como iniciada en la labor investigativa, el método etnográfico utilizado aquí parte de un compromiso en la práctica y la política para representar y dar voz a los grupos afrocolombianos silenciados e invisibilizados por generación y para que como método en el campo de las ciencias sociales de cuenta del aspecto cualitativo de los hechos para intervenir en los procesos mentales, pero también para confirmar aquellas aportaciones de Bourdieu que indican, que la dominación no es numérica sino simbólica ${ }^{7}$.

6 Respecto del misterio de la desigualdad en América Latina, Reygadas que por una parte, los económicas hablan de un dualismo en la estructura productiva, de otra parte, el análisis político ha mostrado la persistencia del clientelismo y el demasiado control que ejercen las élites sobre el Estado; entre tanto, los sociólogos hablan de un colonialismo interno y la escisión entre grupos modernos y tradicionales que explican la desigualdad.

7 A la pregunta, de si las personas de clase social baja no entienden las formas de vanguardia, Bourdieu explica, que por ejemplo en el campo de la televisión, pueden existir canales televisivos que programan espectáculos comerciales tradicionales muy seguidamente, pero estos no los hace más poderosos. Los programas tradicionales en comparación con los de vanguardia y de élite son muy poco frecuentes dice él. En tal sentido, tener el gusto tradicional es una forma de sumisión, ya que se puede ser numéricamente mayoritario y simbólicamente minoritario, que es el caso precisamente de todo el campo del arte. Aunque este es un relativismo dice el autor, persiste la idea, de que las formas más populares del arte vienen a tener valor solo por el hecho de ser plebiscitarias, es decir, una disposición legal tomada por la mayoría. En el fondo puede que en esas formas de arte no operen valores tanto como en otras. Por ejemplo, continúa el autor, existen mercados importantes como el mercado escolástico, en los cuales en unos operan valores y en otros no valen. Su ejemplo más sencillo podría ser el campo del lenguaje en el cual la dominación simbólica se ejercita de manera más visible en la desigualdad de acento (de la transcripción de la entrevista sobre la violencia simbólica sistematizada por Álvaro Moreno y José Ramírez, 2003). 
Así las desigualdades existentes en relación con los grupos afrodescendientes en Colombia, ya lo decía Restrepo (2007), se han producido más por la relación entre discurso y práctica que por el sistema de características físicas que podría separarlos del resto de la población que no se considera como parte del grupo.

No obstante a estas afirmaciones, sabemos que no existen ni posturas ni miradas homogéneas sobre el mundo. En tal sentido, se muestra la relación estadística que tiene "la esclavitud de ayer" con el racismo de hoy para dar cuenta de su relación histórica cuando no de la "falla estructural" introducida por Rodríguez Garavito para dar cuenta, de lo que realmente ha sido la acción del Estado para reconocer y resarcir el olvido, que por generaciones ha afectado negativamente a la población afrocolombiana en materia de políticas públicas, sobre todo en el campo educativo. De modo que aunque pensamos que gran parte de nuestra discusión y reflexión se centra en el análisis y explicación de la producción y reproducción de estas categorías de percepción con las cuales los sujetos afrocolombianos son pensados y ubicados en el espacio social, queremos soportar lo anterior mostrando, que de acuerdo con datos del Ministerio de Educación Nacional, entre los periodos 2010 a 2015, solamente el 0,28\% de los estudiantes matriculados en educación superior universitaria correspondió a población afrodescendiente y en general, en todos los niveles de educación superior para el mismo periodo, esta cifra no supera el $0,25 \%$ respectivamente.

Tabla 1. Porcentaje (\%) de población afrodescendiente en algún nivel de formación superior con respecto al total nacional durante el periodo 2010-2015.

\begin{tabular}{l|c|c|c|c|c|c|c}
\hline $\begin{array}{c}\text { Nivel de } \\
\text { Formación }\end{array}$ & $\mathbf{2 0 1 0}$ & $\mathbf{2 0 1 1}$ & $\mathbf{2 0 1 2}$ & $\mathbf{2 0 1 3}$ & $\mathbf{2 0 1 4}$ & $\mathbf{2 0 1 5}$ & $\begin{array}{c}\text { Promedio } \\
(\mathbf{\%})\end{array}$ \\
Técnica profesional & $\mathbf{0 , 0 5}$ & $\mathbf{0 , 6 0}$ & $\mathbf{0 , 3 6}$ & $\mathbf{0 , 3 5}$ & $\mathbf{0 , 2 6}$ & $\mathbf{0 , 1 8}$ & 0,29 \\
Tecnológica & $\mathbf{0 , 1 4}$ & $\mathbf{0 , 1 1}$ & $\mathbf{0 , 1 1}$ & $\mathbf{0 , 2 0}$ & $\mathbf{0 , 0 9}$ & $\mathbf{0 , 2 5}$ & $\mathbf{0 , 1 5}$ \\
Universitaria & $\mathbf{0 , 3 0}$ & $\mathbf{0 , 2 6}$ & $\mathbf{0 , 2 7}$ & $\mathbf{0 , 3 0}$ & $\mathbf{0 , 2 7}$ & $\mathbf{0 , 2 7}$ & $\mathbf{0 , 2 8}$ \\
Especialización & $\mathbf{0 , 3 0}$ & $\mathbf{0 , 2 8}$ & $\mathbf{1 , 2 8}$ & $\mathbf{0 , 1 3}$ & $\mathbf{1 , 2 9}$ & $\mathbf{0 , 0 6}$ & $\mathbf{0 , 5 7}$ \\
Maestría & $\mathbf{0 , 1 8}$ & $\mathbf{0 , 1 8}$ & $\mathbf{0 , 0 5}$ & $\mathbf{0 , 0 9}$ & $\mathbf{0 , 0 1}$ & $\mathbf{0 , 0 8}$ & $\mathbf{0 , 0 8}$ \\
Doctorado & $\mathbf{0 , 0 0}$ & $\mathbf{0 , 0 0}$ & $\mathbf{0 , 1 0}$ & $\mathbf{0 , 0 8}$ & $\mathbf{0 , 1 4}$ & $\mathbf{0 , 0 6}$ & $\mathbf{0 , 0 7}$ \\
Total & $\mathbf{0 , 2 4}$ & $\mathbf{0 , 2 3}$ & $\mathbf{0 , 2 7}$ & $\mathbf{0 , 2 6}$ & $\mathbf{0 , 2 5}$ & $\mathbf{0 , 2 5}$ & $\mathbf{0 , 2 5}$ \\
\hline
\end{tabular}

Fuente: Ministerio de Educación Nacional (2016).

Ahora bien, a partir de los datos de matrícula en educación superior para el periodo 2010-2015 se construyó un índice simple para observar el ritmo de crecimiento por año en relación con un año base. Así que, partiendo del año 2010, se observa que el crecimiento en la matrícula universitaria en la población total es mayor que en la población afrodescendiente, lo cual en el largo 
plazo generará mayores brechas. Por ejemplo, para el año 2015, el incremento en la matrícula universitaria con respecto al 2010 fue del $37 \%$, mientras que para la población afrodescendiente fue del $21 \%$.

Tabla 2. Índice simple de la matrícula universitaria para el total nacional y la población afrodescendiente.

\begin{tabular}{l|c|c|c|c|c|c|}
\hline \multicolumn{1}{|c|}{ Población } & $\mathbf{2 0 1 0}$ & $\mathbf{2 0 1 1}$ & $\mathbf{2 0 1 2}$ & $\mathbf{2 0 1 3}$ & $\mathbf{2 0 1 4}$ & $\mathbf{2 0 1 5}$ \\
\hline Total & 100 & 110,9 & 116,6 & 124,0 & 131,0 & 137,0 \\
Afrodescendientes & 100 & 95,1 & 104,1 & 123,8 & 114,9 & 121,7 \\
\hline
\end{tabular}

Fuente: elaboración propia con información del Ministerio de Educación Nacional (2016).

Figura 1. Ritmo de crecimiento de estudiantes matriculados en el nivel de formación universitario por año con relación al año base (2010).

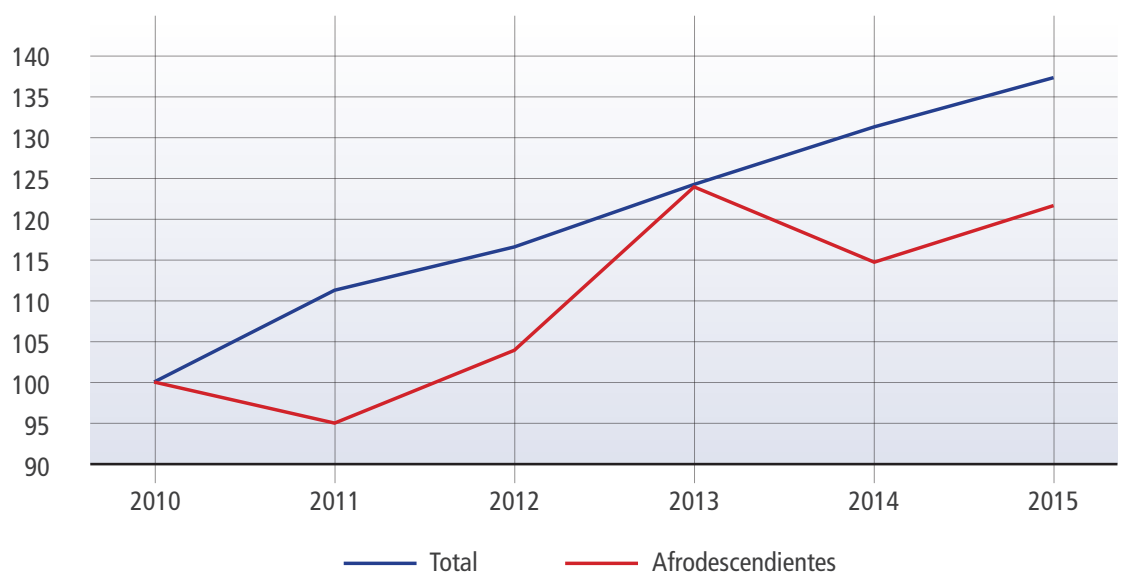

Fuente: elaboración propia con datos del Ministerio de Educación Nacional (2016).

Aunado al bajo crecimiento en la de la población afrodescendiente en relación con el total de personas matriculadas (Figura 1, línea roja), se debe tener en cuenta el tamaño del universo sobre el que se está realizando el cálculo para esta población, que para el año 2015 correspondió al 0,27 \% (Tabla 1) del total nacional de los jóvenes matriculados en nivel de formación universitaria. El efecto de esta baja representación en el total nacional (evidenciándose que es una población minoritaria), es la alta variabilidad en los datos del índice simple para el caso de la población afrodescendiente, lo que pone en evidencia la desigualdad en el acceso a la educación superior universitaria para esta población en Colombia. De este modo, se requerirían estudios con datos desagregados y longitudinales por población, nivel educativo, tipo de universidad, y de ser posible, tasa de egreso versus tasa de ingreso, entre otros indicadores T (Restrepo, 2018). 


\section{Conclusión}

Si queréis entrar en un pentágono y no lo lográis por ninguno de los cinco lados, buscad el sexto.

(ANTiguo PRoverbio Chino) CitAdo POR BECHELloni (1979).

Para concluir se ha tomado a bien reproducir el proverbio chino citado por Bechelloni, en la edición italiana del análisis de los procesos de reproducción de las clases sociales y del orden cultural, al análisis de los procesos de cambio de ese mismo orden. Cuando señalamos que no somos ni sociólogas ni antropólogas, de entrada queremos advertir que hemos asumido el riesgo de abocarnos a un camino desconocido, incierto y con muchas probabilidades de incurrir en el error de iniciadas en un método que puede significar cosas distintas: la etnografía ${ }^{8}$. No obstante, se hace una introducción en el arte y la experiencia de interpretar, explicar y descubrir en el lenguaje aquello que por ciertas costumbres culturales no se dice abiertamente.

En este sentido, se intenta articular las desigualdades descritas en las perspectivas de Bourdieu, Passeron y Reygadas, quienes observan que en el análisis de las capacidades individuales no es suficiente entender la desigualdad social solo en función del reparto de los bienes y recursos, como si estos no siguieran una orientación cultural. Al contrario, para estos autores los bienes y recursos circulan y se intercambian siguiendo reglas específicas, bajo la influencia de instituciones, culturales, económicas y políticas que fluctúan dentro de una dimensión simbólica. Teniendo en cuenta esta dimensión simbólica de la desigualdad, se alude al epígrafe de Mead en el libro Los Herederos: los estudiantes y la cultura de Bourdieu y Passeron (1964), para indicar, cómo a través de las costumbres, ritos y otras creencias se institucionalizan ciertas prácticas de dominación y exclusión, que para el caso que nos compete corresponde a la ideología racista.

Así, la presentación de estas desigualdades es el punto de partida para fundamentar las barreras sociales que impiden la inclusión plena de los grupos afrodescendientes en Colombia, y concretamente dentro del sistema de educación superior universitaria.

Como lo ha señalado Rodríguez (2010), en lo concerniente a las políticas públicas en materia de derechos

Impulsar la política educativa haría parte de esa acción pragmática que le correspondería al aparato estatal orientar en harás de restituir el daño causado a los grupos afrocolombianos como víctimas de la violencia estatal y del desplazamiento forzado como un fenómeno económico y político que ha cambiado la estructura social. (p.438).

8 Restrepo Uribe, Eduardo (2016): Etnografía: alcances, técnicas y éticas. Bogotá: Envión Editores 
En consecuencia, la política educativa debería de nutrirse e incluir con decisión aquéllos indicadores para visibilizar, restituir y hacer seguimiento del daño causado en materia de los derechos que les han sido vulnerados. Si el logro educativo, como lo hacen ver los indicadores en Colombia está asociado a la clase social como factor importante, entonces los efectos particulares del racismo contribuyen a que la inequidad en relación con los grupos afrocolombianos sea mucho mayor. La pregunta que se puede plantear es: ¿cómo es que sucede esto? Y se trae nuevamente a colación el proverbio chino para decir, que si un propósito no se puede lograr por un lado, por las barreras que se puedan enfrentar, entonces habría que buscar, en cuanto sea posible el sexto lado para poder entrar.

Cuando se hace referencia a los efectos particulares del racismo, se intenta hacer la acepción a los elementos que puedan incluir expresiones como "manera borrosa", "formas eufemizadas" y ciertas "ambigüedades", de las referidas por Díaz (2001), donde tiene su asidero el racismo. Como ya lo anotaba Estefanía y el grupo focal de los becarios Martin Luther King, los rasgos culturales estigmatizados son los ejes del auténtico disimulo de las relaciones de poder, del poder de inferiorizar y dominar a los grupos afrocolombianos subordinados en cualquier campo como sucede en el campo de la educación superior universitaria.

Esta alquimia subordinadora e inferiorizadora tiene el poder de triunfar cuando las personas que ocupan posiciones de poder en este campo no son en su mayoría personas afrocolombianas. Entonces, como señala el grupo de becarios, en este espacio social comienza a importar vos quien sos. Y no es que se les olvide el color, sino que lo pasan a un segundo plano, porque entonces lo que importa es quién tiene la plata. Y tal vez en un contexto como el de la educación superior universitaria, sea muy fácil identificar quién tiene la plata. Porque entonces, cuando por las percepciones se te clasifica como negro, ya se sabe y se entiende que eres pobre. Y de este condicionamiento depende también tu clasificación en el espacio social y en cualquier contexto donde un negro se encuentre. Y como parte de las taxonomías que se hacen con relación a lo negro, este artículo producto de mi trabajo de grado es para mí como el sexto lado del pentágono por el cual las mentes y los cuerpos de las personas afro pueden ser reivindicados y escuchados.

\section{Reconocimientos y financiamiento}

Al padre Gonzalo Amaya Otero, sj, que me acogió como una nieta en tierra extranjera.

A los Misioneros Combonianos: padre Rafael Savoia, padre Antonio Arbor, Marco Binaghi, Jose Manuel Salvador, que hicieron posible esta maestría.

A Patrick Klug, mi gran hermano, que me acogió desde siempre sin prejuicios y que hizo posible esta maestría. 
Al padre Massimo Annicchiarico, sj, mi siempre amigo, que en cada momento me hace reír para llevar mejor la carga, y que también hizo posible esta maestría.

A Alicia Méndez, que ha sido como mi madre en el momento en que mi trayectoria por la maestría se hizo más difícil.

A Cecilia Galindo, que me ayudó a dar el primer paso en mi vida profesional y personal.

Al Profesor Pedro Alexander Silva por iser un verdadero profesor!

A Nubia Suárez Sanabria, por compartir sus conocimientos conmigo, por su solidaridad y apoyo con este estudio.

A toda mi Gente afrocolombiana, negra, raizal y palenquera, a las jóvenes y a los jóvenes de grado $11^{\circ}$ que me inspiraron a escribir estas letras.

A las estudiantes y los estudiantes de las universidades que me apoyaron compartiéndome sus trayectorias por la universidad.

A Luca Kiser, por su estipendio para la maestría.

A Matthias Dissle Schäfer, por su estipendio para la maestría.

A Teresa Sprenger, por darme la oportunidad de tener un empleo y por valorar mis habilidades.

A Coline David, por valorar mis esfuerzos y apoyar mi lucha con los jóvenes afrocolombianos.

A Giuseppina Di Martino, por su estipendio para la maestría.

A Christina Scholz, por su estipendio para la maestría.

A Simona Tumino, por su estipendio para la maestría A Giuseppe Velardi por su estipendio para la maestría A Sophie Mohr, por su estipendio para la maestría.

A Anyela Pérez García y Stephanie Trujillo, por enseñarme a soñar y volar más lejos de lo que nunca imaginé.

A Yurary Marcela Céspedes, por ser una buena compañera y por los momentos compartidos durante la maestría.

Disponibilidad de datos y materiales: Repositorio Pontificia Universidad Javeriana Contribución de autores: La contribución de los estadistas Nubia Suárez, Nallydis Hernández y Álvaro Suárez, permitió elaborar un índice simple para observar con datos estadísticos el crecimiento en la matrícula de los jóvenes afrocolombianos durante el periodo indicado. 
Aprobación ética y consentimiento de los participantes: aprobado por el tutor del trabajo de grado y firma del consentimiento informado de los jóvenes de grado $11^{\circ}$ de la institución educativa donde se realizó el estudio.

Conflicto de intereses: NO existe ningún conflicto de interés de ninguna naturaleza.

\section{Referencias}

Andrade, B. y Andrade, C. (2012). Acciones afirmativas (políticas educativas internar delas universidades publicas colombianas paralos afro-colombianos). Revista Inciso, Vol. 13.

Bourdieu, P. y Passeron, J.C. (1979). La Reproducción: elementos para una teoría del sistema de enseñanza. Barcelona: Editorial Laia.

Bourdieu, P. y Passeron, J.C. (1964). Los Herederos. Buenos Aires: Siglo XXI.

Bourdieu, Pierre.1988. La distinción: criterios y bases sociales del gusto. Madrid: Taurus

Cárdenas, J., Ñopo, H. y Castañeda, J. (2012). Equidad en la diferencia: políticas para la movilidad social de grupos de identidad. Documento CEDE, 39.

Caston, B.1996. La sociología de Pierre Bourdieu. Universidad de Granada

Díaz, R. (2001). Esclavitud, región, y ciudad. Sistema esclavista urbano-regional en santa fe de Bogotá 1700-1750. Bogotá: CEJA.

Fanon, F. (2010). Piel negra, máscaras blancas. Madrid: Akal.

Gutiérrez, A. (2011). Pierre Bourdieu las estrategias de la reproducción social. Buenos Aires: Siglo XXI.

Melo, L., Ramos, J. y Hernández, P. (2014). La Educación Superior en Colombia: situación actual y análisis de eficiencia. Borradores de Economía, 808.

Ministerio de Educación Nacional (MEN). (2016). Resumen estadístico de educación superior: subdirección de desarrollo sectorial. Bogotá: MEN.

Morales, G. (2008). Metamorfosis del paradigma del reconocimiento: del racismo biológico al racismo cultural. Universitas. Revista de Filosofía, Derecho y Política, 7, 71-85.

Moreno, Á. y Ramírez, J. (2003). Pierre Bourdieu: introducción elemental. Bogotá: Editer Estrategias Educativas Ltda. 
Procuraduría General de la Nación.(2006). El derecho a la educación. Procuraduría delegada para la prevención en materia de derechos humanos y asuntos étnicos. Giro Editores Ltda.

Restrepo, E., Walsh, C. y Vich, V. (2010). Stuard Hall: Sin garantias: Trayectorias y problemáticas en estudios culturales. Bogotá: Universidad Javeriana Envión editores, Instituto de Estudios Sociales y Culturales, Pensar.

Restrepo, E. (2007). Acción afirmativa y afrodescendientes en Colombia. Recuperado de: http://www.ram-wan.net/restrepo/documentos/accionafirmativa.pdf.

Restrepo, A. (2018). Equidad en educación superior: variaciones en torno a sus itinerarios y tensiones. Educación Crítica y Emancipación, (1), 31-60.

Reygadas, L. (2008). La Apropiación: destejiendo las redes de la desigualdad. México: Anthropos Editorial.

Rodríguez, C, Alfonso, T. e Cavelier, I. (2009). Raza y derechos bumanos en Colombia: informe sobre discriminación racial y derechos de la población afrocolombiana. Bogotá: Ediciones Uniandes.

Rodríguez, C. (2010). Más allá del desplazamiento: políticas, derechos y superación del desplazamiento forzado en Colombia. Bogotá: Ediciones Uniandes.

Rodríguez, M. (2016). Tensiones para la inclusión real en la educación superior: la institucionalidad desde distintas perspectivas: el caso del programa de admisión especial para estudiantes negros, afrocolombianos, palenqueros y raizales de la Universidad Nacional de Colombia. Buenos Aires: CLACSO.

Subirats, M. (1979): Introducción a la edición castellana. La Reproducción. Pierre Bourdieu y Jean-Claude Passeron. Barcelona: Editorial Laia.

Tilly, C. (2000). La desigualdad persistente. Buenos Aires: Editorial Manantial. 